\title{
FATAL DAMAGE TO THE BRAIN BY EPILEPTIC CONVULSIONS AFTER A TRIVIAL INJURY TO THE HEAD
}

\section{BY}

\author{
J. M. SMALL and A. L. WOOLF
}

\author{
From the Midland Centre for Neurosurgery, Smethwick, Staffordshire
}

The susceptibility of the brain in childhood to anoxic damage during epileptic convulsions is still not yet widely recognized in spite of the pathological studies by Scholz, summarized in his recent monograph on the subject (1951). Two years ago Meyer and his collaborators reported the case of a boy aged 9, who, following status epilepticus developing in the course of Still's disease, was found to be demented, and subsequently suffered from various types of epileptic seizure (Meyer, Beck, and Shepherd, 1955). Post-mortem examination showed widespread damage to the brain apparently due mainly to circulatory and anoxic disturbances associated with the first status epilepticus.

In the case we report below, widespread cerebral lesions, also apparently anoxic in origin but in an appropriately fresh state, were found three days after status epilepticus, which followed a trivial injury to the head after a lucid interval of 24 hours.

\section{Case Report}

The patient, a boy aged 13 years, was one of five children, all, including himself, in good health and without any history of fits.

The patient had had no previous illness apart from measles as a baby, On the evening of July 7 , he was fielding whilst playing cricket with two other boys, when his attention was momentarily diverted by someone calling to him. At that moment, he was struck by the ball, which was of cork, full on the forehead. The ball had come directly from the bat without hitting the ground. He fell over backwards, but picked himself up and went on playing as though unaffected. Later he went home with his friend, passed a normal night and the next day went to school. He behaved normally at school, but boasted about the bruise on his forehead. At 4.15 p.m. he returned home, carried out a shopping errand and seemed in every way normal until 6.15 p.m., when his mother noticed that he appeared to be "unconscious". A few moments later, he got up from his chair with his eyes shut and picked up a piece of bread from the table, put it in his mouth, then took it out and made as though to throw it in the fire. He then had a fit, all limbs convulsing, and became unconscious. He was taken to West Bromwich and District Hospital, and was admitted under Mr. J. H. Kirkham at 7 p.m. He was semiconscious but rousable, the pupils were equal and reacted to light, and except for a decrease in the right knee jerk, all the tendon reflexes and abdominal and plantar responses were normal. The blood pressure was $110 / 70 \mathrm{~mm}$. $\mathrm{Hg}$.

Between 7 p.m. and 10 p.m. he vomited once. At 6 a.m. the next morning, he had a further fit lasting six minutes. The arms and legs twitched and he then went very blue. The pupils remained normal, the right plantar response became extensor and his pulse was 82 per min. Half an hour later, he was rousable again and had a good colour. Later in the morning, he recognized the surgeon who had treated him previously for an implantation dermoid, and spoke coherently to his mother. At 10.5 p.m. that evening, he had a very severe fit lasting three-quarters of an hour. We are indebted to Dr. F. G. Duffy for the following account of the fit:-

"I was asked to see the patient at midnight when he had already been in status epilepticus for 35 minutes. When I saw him, he was still convulsing, was moderately cyanosed, and there was very marked respiratory embarrassment. Even from the foot of the bed, it was obvious that the respiratory passages were very obstructed and moist, and he appeared to have vomited during the course of the fit.

I injected $5 \mathrm{ml}$. ( $\frac{1}{4} \mathrm{~g}$.) of $5 \%$ thiopentone intravenously. Within a few seconds the fit was controlled, but the patient remained slightly cyanosed and his chest still sounded 'very wet'. Pharyngeal toilet was performed and the patient was put back on oxygen, this time with a properly fitting mask and reservoir bag.

About 20 minutes after the fit had been controlled, twitching recommenced in the left forearm and hand, $1 \frac{1}{2} \mathrm{ml}$. of thiopentone was given intravenously, and the twitching stopped immediately. Paraldehyde, $5 \mathrm{ml}$., was given intramuscularly and, approximately 30 minutes later, scopolamine, g. 150, was given."

He had a further fit at 4.30 a.m. the next morning, after which his pulse rose to 130 per minute, and the right eye turned inwards. The next day, July 10, he was still unconscious, but could be roused and spoke a single sentence to his mother on one occasion only. He was incontinent. The pupils were normal, but both plantar responses were now extensor. The abdominal reflexes were very sluggish, and the knee and ankle jerks absent. He slept fairly well during the night, but was restless at times. He took sips of water. After 11.30 a.m., his 
condition deteriorated and at 12.30 p.m. he became unconscious with a rising pulse (to 140 per minute) and respiration (to 40 per minute rate). The pupils remained equal and reacted to light. At 6 p.m. he was transferred to the Midland Centre for Neurosurgery, where he was seen to be lying still, but showed some twitching of the right arm. On supraorbital pressure, he moved the right face, arm, and leg, and the right arm and leg on pin prick. He did not answer the spoken word. There was slight neck stiffness, but Kernig's sign was absent. There was internal strabismus. The margin of the left optic disc was swollen and obliterated. The left pupil was larger and more reactive to light than the right. There was a left facial paralysis. The limbs were flaccid and there was generalized areflexia. The left plantar response was extensor.

Two frontal and two temporal burr holes were made under local anaesthesia. The intracranial pressure was normal or low. No extra- or subdural blood was found. Cerebrospinal fluid welled up satisfactorily. Air was instilled into each temporal horn and radiography showed a general shift from right to left with downward compression of the right lateral ventricle more anteriorly than posteriorly. A further right fronto-temporal burr hole was made, and the brain needled and found to be soft. No blood was encountered.

Later that evening there was a continual movement of the right hand and wrist like a slow-motion pill rolling, the pulse and respiration rate rose, and he died at 11.30 p.m.

\section{Post-mortem Examination}

The necropsy was carried out within 12 hours of death. The body was well nourished and normally developed for the age. The skin on the side of the bridge of the nose as far as the angle of both eyes, together with that over the central part of the forehead, was greenish-yellow. Over the centre of the left deltoid, there was a partially healed B.C.G. vaccination sore.

On reflecting the scalp, two burr holes were seen just above the forehead, $6 \mathrm{~cm}$. above the bridge of the nose and $1.5 \mathrm{~cm}$. on either side of the mid-line. There was a very small collection of blood around the left frontal burr hole. Apart from a very few small haemorrhages under the fasciae of both temporalis muscles and under the periosteum over the crown of the head, no abnormality was seen.

The skull and cranial contents were removed in toto. Bone and dura were removed over the frontal and occipital lobes to allow access of formol saline. After two days, the frontal and occipital poles were removed to allow formol saline to enter the deeper parts of the brain. In this way post-mortem damage to the unfixed brain was prevented.

The left axillary and cervical posterior triangle lymph glands were dissected out and appeared quite normal. The thymus appeared a little large. There were a few millilitres of clear yellow fluid in the pericardium and there was a small transparent nodule on the cordae tendinae of the posterior cusp of the mitral valve. The lungs were fixed undissected by intratracheal formalin, and no abnormality was observed on subsequent slicing.
The suprarenal glands had little lipoid, but were otherwise normal. The kidneys were congested. There was no abnormality in the alimentary tract.

\section{Histology of the Extracranial Organs}

Skin from Ulcerated Area at Site of B.C.G. Vaccination over Left Deltoid.- The ulcer was lined by granulation tissue in which lymphocytes predominated. There were, however, also many macrophages. The walls of the small vessels were thickened and occasionally the lumen was occluded by eosinophil material, probably thrombus. Deep to the granulation tissue, there was a thick layer of dense collagenous tissue, beneath which a few collections of inflammatory cells were seen in the subcutaneous fat.

Left Supraclavicular Lymph Node.-Sinus catarrh was marked with frequent macrophages in the sinuses.

Liver.-There was marked oedematous thickening of the collagen in the portal tracts and around the central veins and sinusoids. In some areas, and especially around the central veins, the liver cells were degenerated to the point of necrosis.

Kidneys.-Apart from oedema of the collagen around some of the arterioles, the only abnormality consisted of approximately circular deposits of an amorphous, haematoxylinophilic material, mostly in relation to the cells of the convoluted tubules, but also occasionally in relation to a glomerular tuft or basement membrane. The deposits were approximately twice the size of a red blood cell.

Heart Muscle.-The heart muscle was normal except $\varnothing$ for amorphous deposits similar to those seen in the kidney. The deposits mostly lay against, or upon, a muscle fibre.

The suprarenal gland was normal.

\section{Dissection of the Brain after Fixation}

The only abnormality on the external surface of the brain was a broad, bean-shaped area of subpial haemorrhage, $1.8 \mathrm{~cm} . \times 0.8 \mathrm{~cm}$. in diameter, at the left frontal pole. On section, the underlying cortex was seen to be replaced almost completely by small, partly confluent haemorrhages. It was considered that this lesion had been caused by coagulation of a small vein where the overlying burr hole was made. The posterior half of the right superior parietal convolution was also slightly broadened.

On coronal section, the white matter of the right frontal lobe was shrunken with a greyish discoloration and increased prominence of vessels both in the grey and white matter (Fig. 1). On the right side, just deep to the cortex in the depths of the inferior frontal sulcus, was a lens-shaped haemorrhage, $0.5 \mathrm{~cm}$. long. A haemorrhagic needle track extended through the centrum semiovale of the right cerebral hemisphere into the anterior part of the putamen.

Section through the posterior half of the right superior parietal convolution revealed a crescentic, slit-like cavity, embracing the grey matter of that part of the gyrus which lay in the depth of the sulcus (Fig. 2). Further 


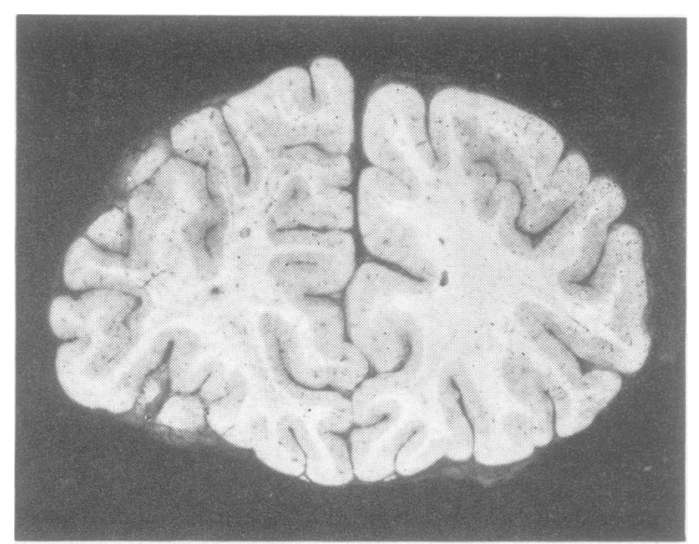

Fig. 1.-Coronal section through the frontal lobes. The right frontal lobe is on the left in the photograph. It shows shrinkage and slight greyish discoloration of the white matter, which contains a small lens-shaped haemorrhage.

forward, the cavity became more superficial, splitting the cortex into two parts. The method of fixing the brain in situ made it impossible that this cavitation could have been produced after death.

No tentorial grooving of either hippocampal gyrus was observed at the level of Ammon's horn. Further forward at the level of the amygdaloid nuclei, there was certainly no grooving on the left, while on the right, the greater part of the hippocampal gyrus was so softened that its surface no longer presented a stable outline.

In all other respects, the brain appeared normal to the naked eye.

\section{Histological Techniques Used}

Blocks were taken for celloidin embedding from the left and right frontal and temporal lobes, the right occipital lobe, and the basal ganglia of both sides at two levels, the region of the dorsal part of the central sulcus on both sides, the mid-brain, pons, and medulla with adjacent foliae of cerebellum. Sections were stained by Nissl, Woelcker for myelin, and van Gieson. Frozen sections from the right occipital and frontal lobes were also stained with Marchi-trichrome, Scarlach $\mathbf{R}$, the Takya-Siko modification of Kulschitsky-Pal and Holzer for glial fibres. Large blocks of one hemisphere were also taken from the frontal and occipital lobes, from both hemispheres at the level of the lentiform nuclei, and from the left temporal lobe and part of the right at the level of the pulvinar. These blocks were impregnated by the Swank-Davenport modification of Marchi's method.

\section{Histological Findings}

Distribution of Nerve Cell Changes.-The distribution and severity of the nerve cell changes are indicated in Fig. 3. Briefly summarized, the cortex of the right cerebral hemisphere was severely affected in all areas studied, except for a small area of the dorsal-most convolution of the occipital lobe. The frontal and temporal lobes were most affected and the cortex on either side of the fissure of Rolando was least affected. There was loss of nerve cells in the affected areas varying from an outfall of isolated nerve cells, through palings, to complete loss of nerve cells from several adjacent gyri (Figs. 4 and 5). Where nerve cell loss was severe, there was a marked hypertrophy and proliferation of the microglia with frequent cells showing mitosis. The astrocytes showed activated nuclei, but had not proliferated. The surviving nerve cells in the cortex appeared normal. No lipoid was seen within the microglia, except for one small group of nerve cells showing ischaemic changes in the right occipital lobe. The amygdaloid nucleus suffered almost total loss of nerve cells. The left hemisphere was much less severely affected, but Ammon's horn was markedly involved on both sides (Fig. 6). On the right side, there was a total loss of nerve cells from the vulnerable $\left(h_{1}\right)$ Sommer's sector and severe degeneration of the nerve cells in the endfolium $\left(h_{3}\right)$ and subliculum. The nerve cells in the resistant $\left(h_{2}\right)$ sector were well preserved, though a few of those nearest to the ventricle showed severe cell change and were undergoing neuronophagia. There was a small island of intact cortex occupying the dorsal half of the hippocampal gyrus, but the cortex of the rest of this gyrus and of the other temporal gyri was severely affected (Fig. 5). The left Ammon's horn showed similar changes to the right, except that there were still many recognizable though degenerating nerve cells in the endfolium. The rest of the left temporal cortex, including the hippocampal cortex, was slightly affected or unaffected. In the thalamus, the right anterior and the anterior part of the dorsomedial nucleus were moderately severely affected (Fig. 7). The posterior part

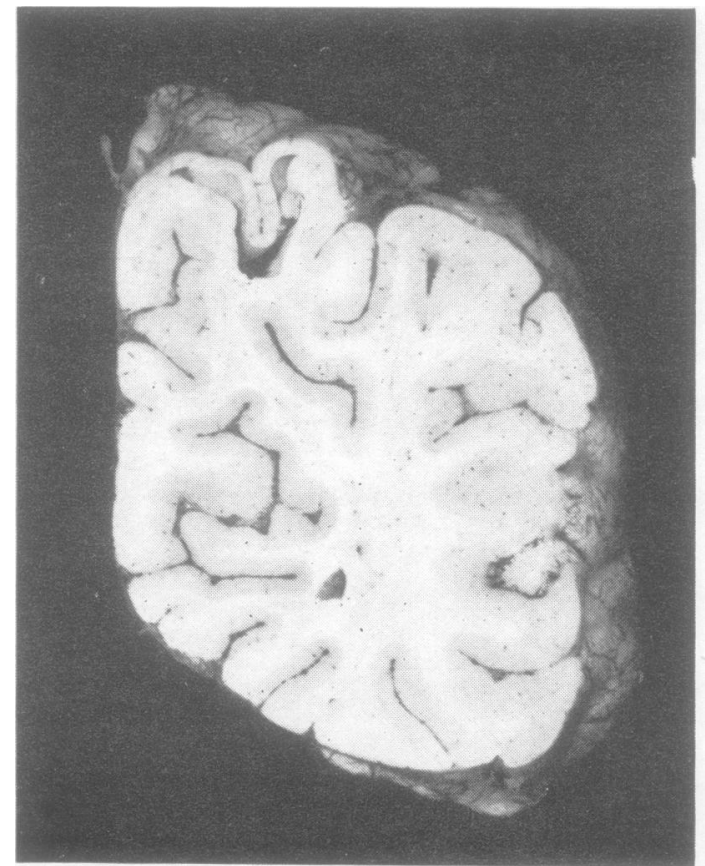

FIG. 2.-Right occipital lobe showing cavity embracing cortex of superior parietal convolution. 

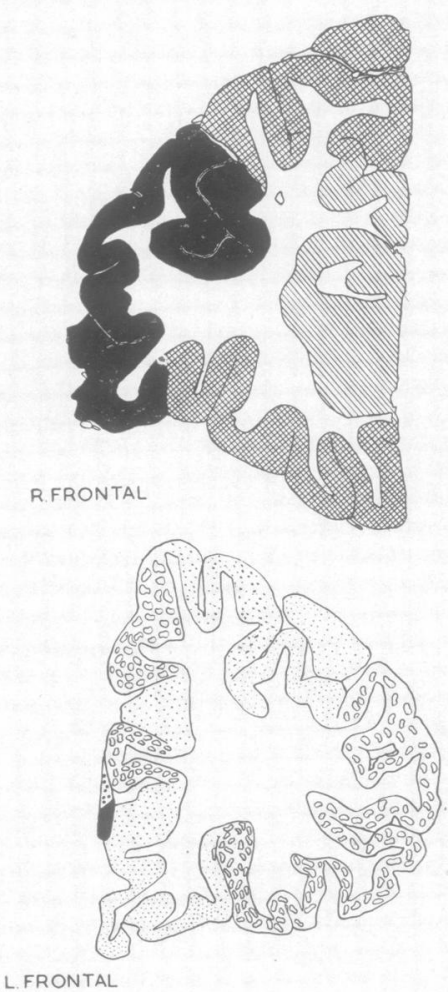

Normal cortex.

Outfall of single nerve cells.

Small palings.

Moderately severe nerve cell loss or degeneration.

Very severe destruction of nerve cells, only 1-4 cells remaining in high-power field.

Complete loss of nerve cells.

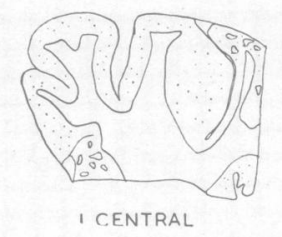

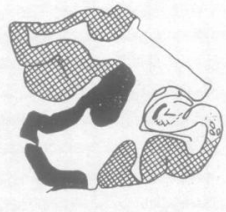

R.TEMPORAL

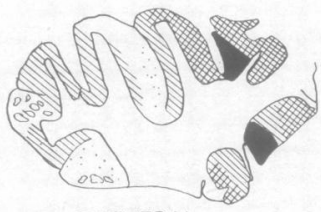

R. CENTRAL

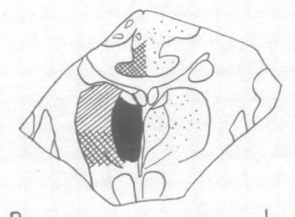

口

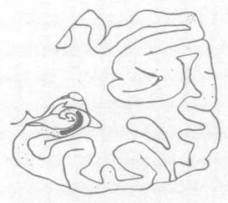

L.TEMPORAL

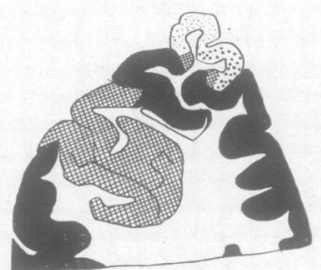

R.OCCIPITAL

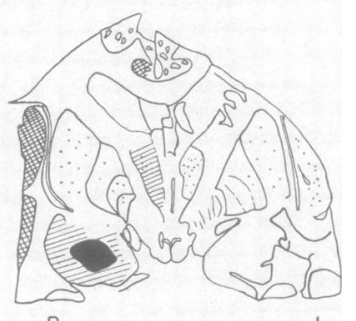

$R$

힘

FIG. 3.-Diagrams illustrating distribution and severity of degeneration of nerve cells.

of the left dorsomedial nucleus showed an outfall of occasional nerve cells only, while on the right side, there was total loss of nerve cells from this nucleus. The centromedian nuclei suffered with the dorsomedial nuclei. The two nuclei laterales posterior were equally affected, the nerve cells showing homogenizing cell changes without any marked outfall. Both red nuclei were severely affected, many nerve cells having disappeared and many others appearing as ghosts with oligodendroglial satellitosis. The subthalamic nuclei, lateral geniculate bodies, and substantia nigra were unaffected. The globus pallidus and putamen were not greatly altered on either side, though an occasional nerve cell in the former and the majority of the large nerve cells in the latter, showed severe nerve cell changes. The caudate nuclei were unaffected. No changes were seen in the cranial nerve nuclei, the inferior olives, or any other nucleus in the brain-stem. The cerebellum (both lateral lobes and vermis) showed patchy degeneration (pale staining and shrinkage) of Purkinje cells with amoeboid and mitotic microglia spread along the dendrites of the degenerating cells (Fig. 8). The one dentate nucleus examined showed a minority of pale-staining degenerate nerve cells.

Changes in the White Matter.-There was a generalized diffuse pallor of myelin staining throughout the lobes of the cerebral hemispheres, but the myelin of the deep white matter, e.g., internal capsule and corpus callosum, was well preserved (Figs. 9 and 10). The most severely 


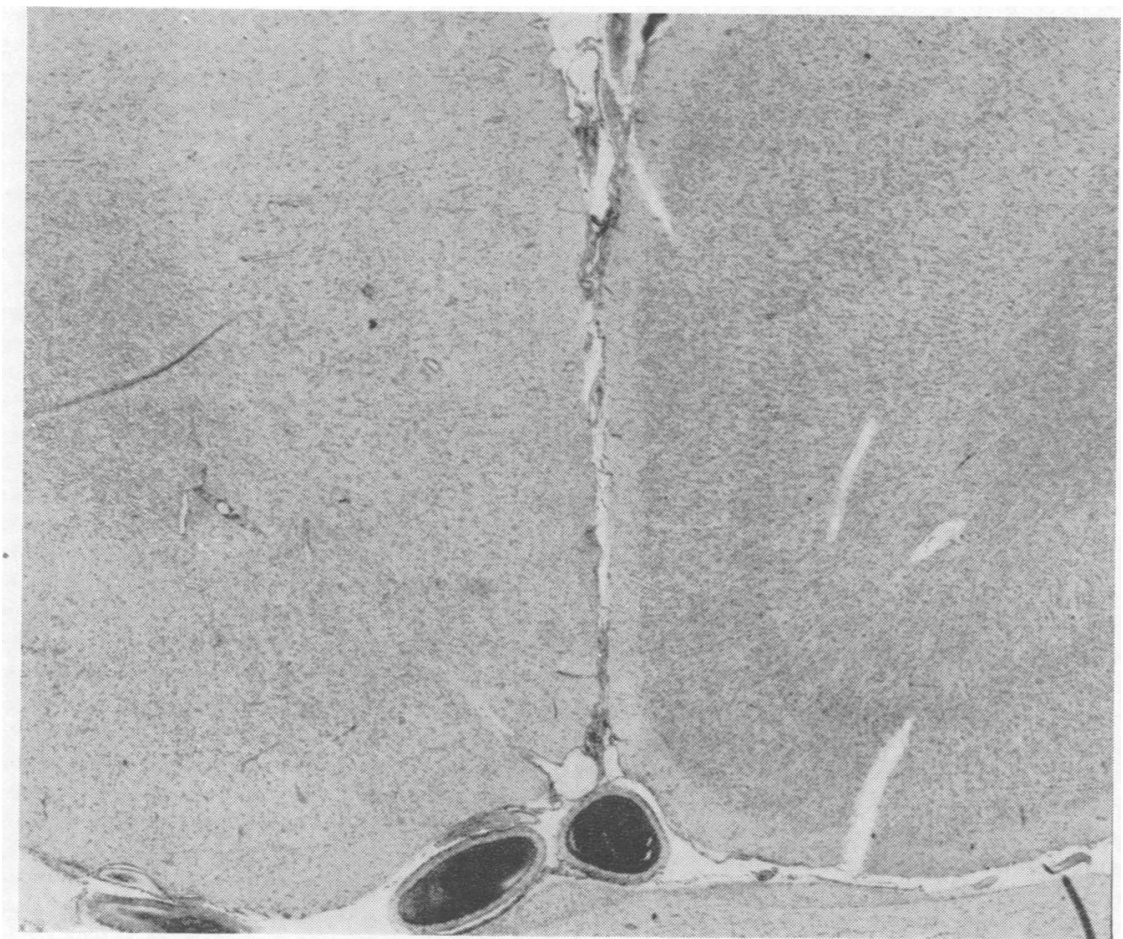

FIG. 4

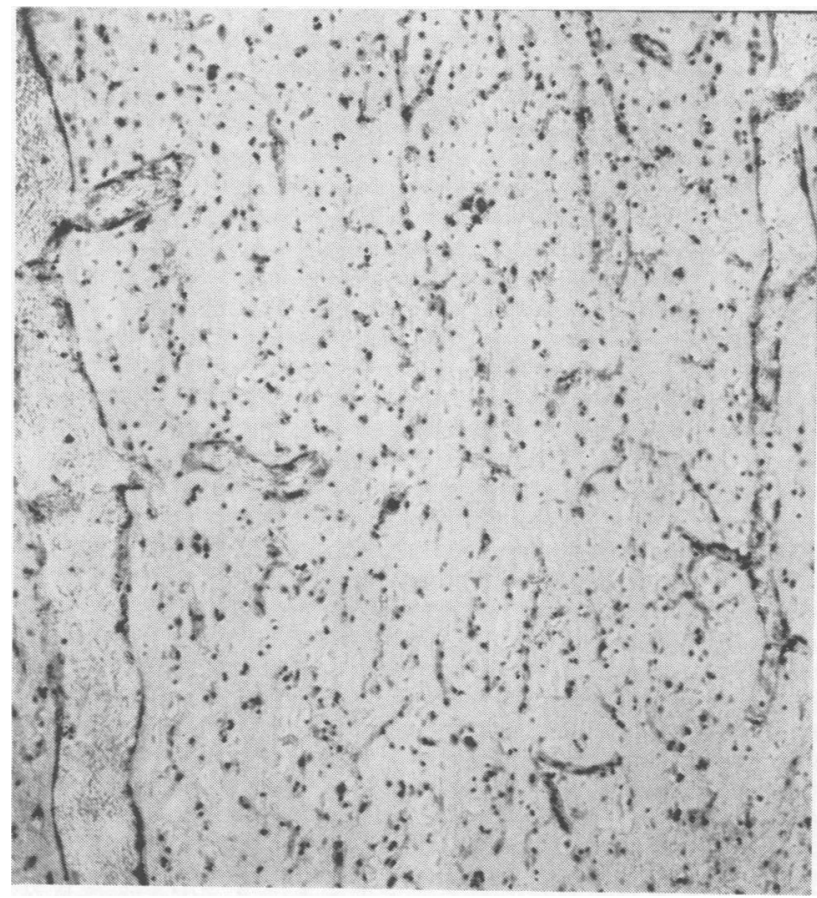

FIg. 4.-Right and left cingulate gyri. The right cingulate gyrus (on left) shows complete loss of the normal lamination due to total disappearance of nerve cells. The left gyrus appears normal. Nissl.

FIG. 5.-Cortex of right gyrus fusiformis (temporal lobe) showing severely degenerated nerve cells laying in vacuoles; the microglia is proliferating and there is extreme dilatation of vessels with activation of endothelial cells and formation of phagocytes. Nissl.

Fig. 6.-Right Ammon's horn and dorsal half of hippocampal gyrus, showing preservation of nerve cells in the resistant $\left(h_{2}\right)$ sector and destruction of cells in Sommer's $\left(h_{1}\right)$ sector, endfolium, and subiculum. The cortex of the dorsal half of the hippocampal gyrus is intact (HG). The cortex of the ventral half of this gyrus, as well as of the other gyri of the right temporal lobe showed very slight loss of nerve cells. Nissl.

FIG. 7.-Right dorsomedial nucleus showing degeneration and neuronophagia of nerve cells. Arrow indicates microglial cell in mitosis. Nissl.

FIG. 8.-Cerebellum showing microglia spread out along dendrites of degenerating Purkinje cells. Arrow indicates microglial cell in mitosis. Nissl.

FIG. 5 


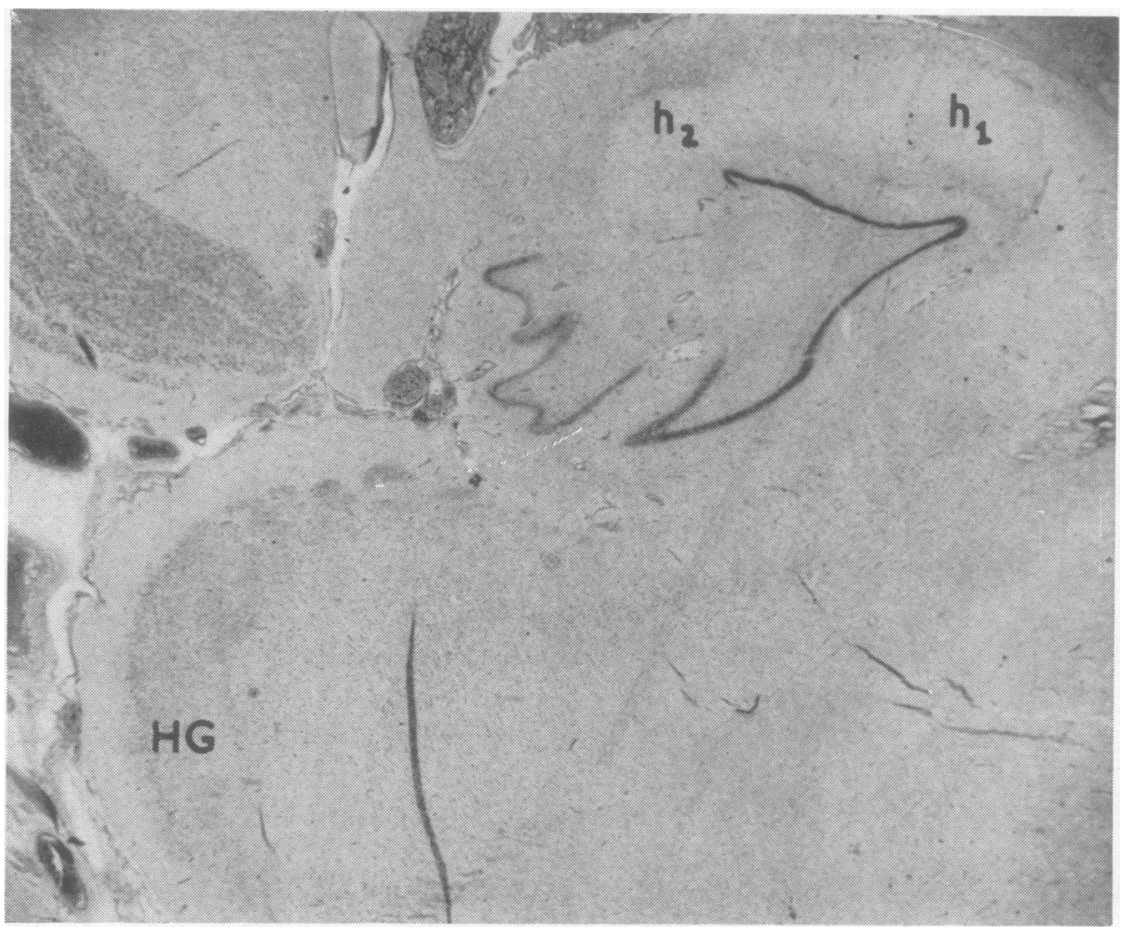

FIG. 6

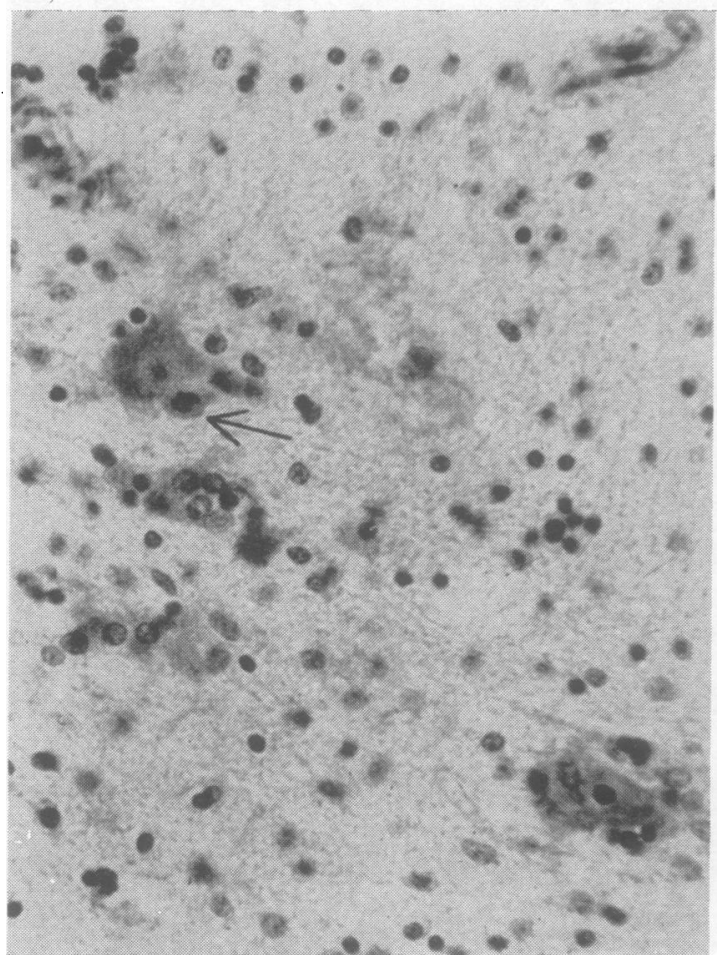

Fig. 7

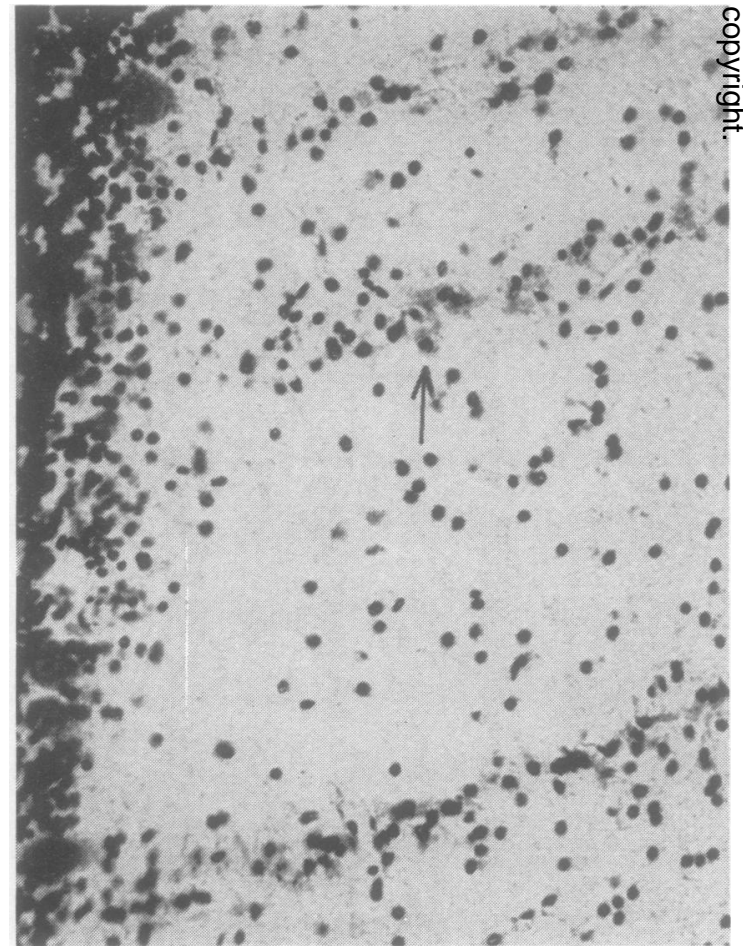

FIG. 8

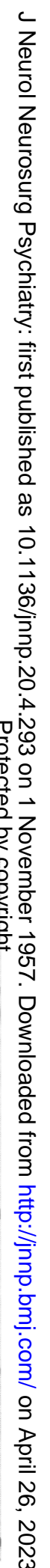

옹으

¿

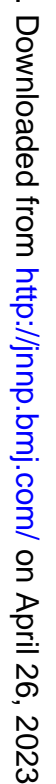


Fig. 9.-Right occipital lobe showing severe pallor of myelin staining in centrum semi-ovale and crescentic cavity embracing cortex of superior parietal convolution. Woelcker.

FIG. 10.-Coronal section through basal ganglia showing normal staining of myelin of corpus callosum and internal capsule. Woelcker.

Fig. 11.-Right frontal lobe showing severe, and in places total, pallor of myelin staining. Woelcker.

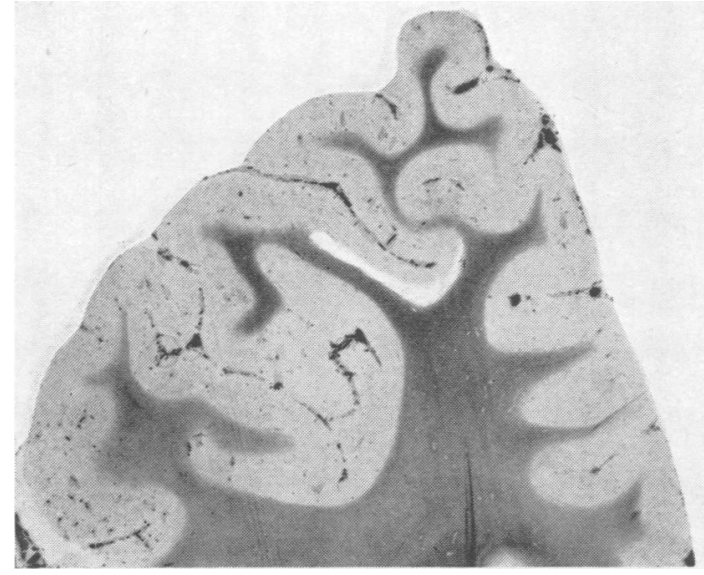

FIG. 9

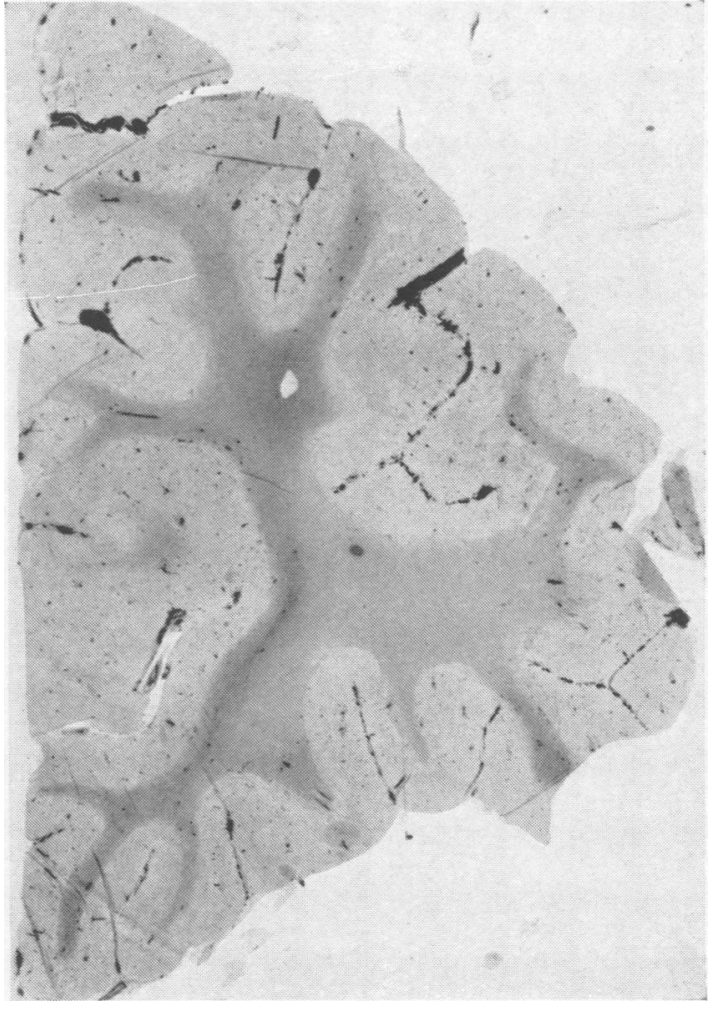

Fig. 11

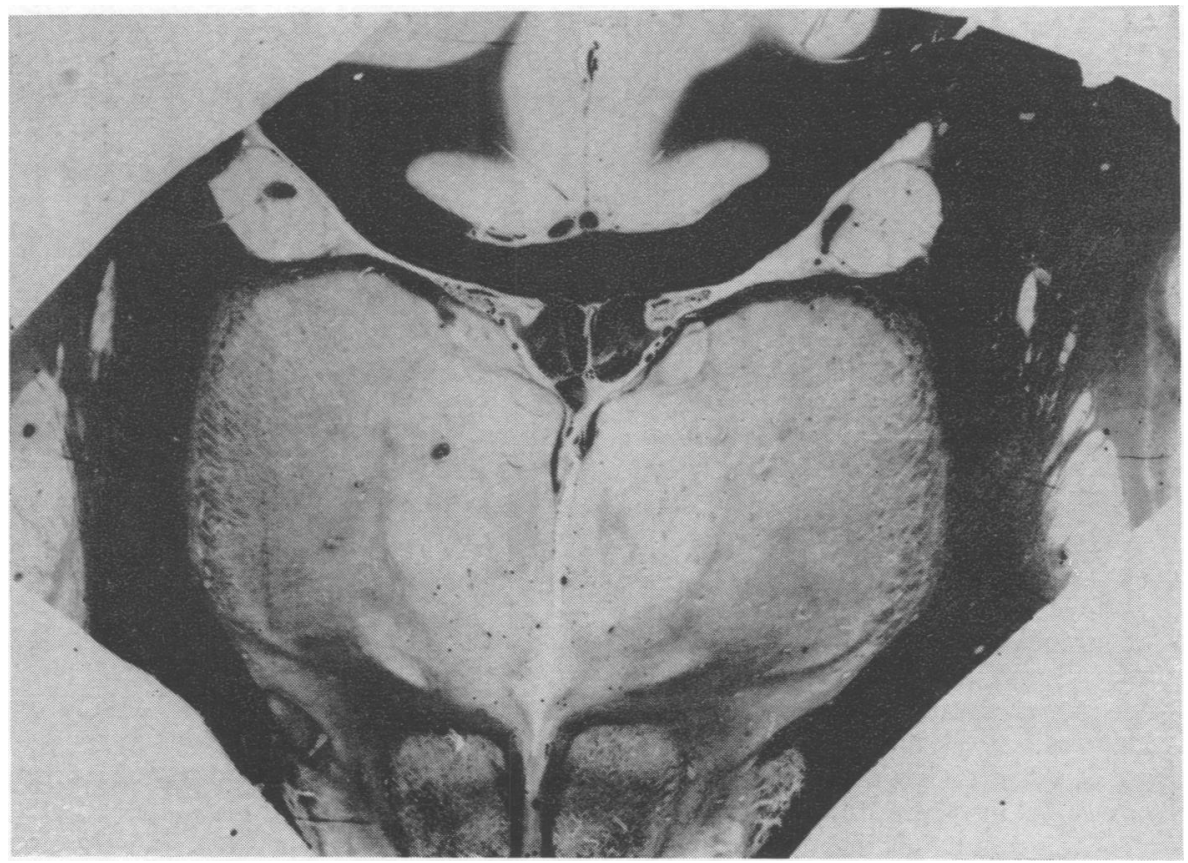

Fig. 10 
affected white matter was that of the right frontal lobe (Fig. 11), which showed absolute lack of staining deep to the inferior frontal convolutions. The best preserved myelin of the centrum semi-ovale was that around the left fissure of Rolando. Both temporal lobes and the left frontal lobe were severely affected, but not so severely as the right frontal lobe. The optic radiations stained more deeply than the rest of the white matter of the temporal lobe. The corpus callosum was not affected. There was slight pallor of the myelin of both middle cerebral peduncles.

In spite of the severity and extensiveness of the degeneration of myelin, there was no evidence of the products of degeneration. Scharlach $\mathbf{R}$ preparations from severely affected areas showed only a doubtful increase in anisotropic lipoid in the occasional phagocytes around the vessels in the white matter. A small portion of the white matter from the right cerebral hemisphere, taken within a month of fixation, showed numerous Marchi-positive myelin sheaths, but this was probably an artefact. Large blocks, taken after a longer period of fixation, showed only scattered granules of Marchi-positive material, none of which were intracellular. The glia was little affected in the white matter except in the right frontal lobe where there were numerous amoeboid microglial cells, many of which were in mitosis.

\section{Discussion}

The main interest in this case is in the pathogenesis of the fatal damage to the brain, which was so little anticipated in view of the triviality of the blow to the head.

Since the patient was behaving entirely normally until the time of his first convulsion about 24 hours after he was hit on the head, it seems unlikely that any extensive damage to the brain immediately followed the blow. On the other hand, the state of the degeneration of nerve cells, especially in the cortex of the frontal and occipital lobes from which most of them had disappeared, was such that it must have begun not less than two, and probably three, days previously. The changes in the dorsomedial nucleus of the thalamus appeared a little more recent. It seems very likely, therefore, that the neuronal degeneration began after the first convulsion, but did not reach its full development until after the attack of status epilepticus on the second night, after which coherent speech was lost and drowsiness deepened into unconsciousness.

The lesions are striking both in their distribution and in the structures affected. In both respects, they are typical of anoxia. Cerebral and cerebellar cortex, Ammon's horn, and especially Sommer's sector, are well known for their sensitivity to lack of oxygen. Lindenberg (1955) has attempted to explain the special vulnerability of Sommer's sector in terms of compression of the long, but not the short, arteries of Ushimura against the tentorium as a result of raised intracranial pressure. In a similar way, he would explain the escape of the hippocampal cortex and destruction of the nerve cells in the adjacent temporal convolution as a result of compression of temporal branches of the posterior cerebral artery. It should, therefore, be stressed that at operation the dura did not appear to be tense, and the ventricular C.S.F. was not under increased pressure. Dissection of the brain, fixed in situ, showed no herniation of the hippocampal gyrus at the level of Ammon's horn. After dissecting brains in which the arteries had been injected with neoprene before fixing in situ, it has seemed to us unlikely that the long arteries could regularly be occluded by pressure of the tentorial edge, while the adjacent short arteries should equally regularly escape. The selective vulnerability of the $h$ sector of Ammon's horn, in our opinion, still requires an explanation.

The recent recognition and significance of degeneration of the amygdaloid nucleus as a postepileptic phenomenon has been discussed by Meyer et al. (1955) and will not be discussed further here. Attention will only be drawn to its extreme severityo on the right side in the case reported here.

In all the areas affected, the nerve cells alone had degenerated, although the numerous mitoses in the $=$ microglia demonstrated the stimulus that had beerp given to this tissue by the degenerating nerve cells and their processes. This is the picture of "selectives parenchymal necrosis", the name given by Scholz. to this most characteristic effect of anoxia. It remains to postulate the way in which anoxia can arise in the course of an epileptic fit or status epilepticus. Scholz refers to increased oxygen demands and vasomotor disturbances, but it seems to us that the cerebral anoxia may be part of a more generalized anoxia. The necrosis of the liver in our case supports this conception. The cyanosis and respiratory embarrassment which accompany every attack of major epilepsy must inevitably produce some anoxia, and it is surprising that damage to the brain is not more frequently discovered. Even if this is the primary cause of the anoxia, vasomotor disturbances and differential oxygen consumption and sensitivity to oxygen deficit will still be important in determining the topography of the lesions. It is also probable that those nerve cells discharging most actively in a fit are more likely to degenerate in the presence of anoxia. The changes in the nerve cells do not require further comment; they are typical of anoxia. The asymmetry of the cerebral involvement did not find expression in descriptions available of the fits, but it is possible that the margin between an anoxia, sufficient to affect nerve cells at all and that adequate to cause fatal degeneration, is very small, so that 
both hemispheres may in fact have suffered a comparable anoxia. The pallor of myelin staining in the centrum semi-ovale uas more symmetrical and, though typical of oedema, may in fact have been an indirect effect of anoxia, since anoxic damage to the endothelium of blood vessels may readily be conceived to result in increased permeability and oedema.

The complete absence of the products of breakdown of myelin in areas showing very marked pallor of myelin staining is probably due to the short time elapsing between the convulsions and death. Nevertheless, even where survival is more prolonged, oedema characteristically produces a pallor of myelin staining in which breakdown products and compound granular corpuscles are inconspicuous (Greenfield, 1939; Scholz, 1949; Woolf, 1954). The absence of stainable perivascular exudate is also not incompatible with oedema and merely indicates that the increase in vascular permeability, though widespread and prolonged, was insufficient to allow the passage of the protein constituents of the plasma (Scholz, 1949). The relatively intact state of the large commissural tracts is a well recognized finding in cerebral oedema (Jaburek, 1936).

It is interesting that not only in the cortex, but also in the white matter of the right frontal lobe, were the changes most advanced as regards the microglial response, suggesting that the other areas may have been affected after the severe attack of status epilepticus on July 9 . The right occipital lobe was also severely affected, and it is important to note that crescentic cavities at the juction of cortex and white matter and embracing a sulcus may result from partial sequestration of cortex deprived of nerve cells, though without degeneration of the interstitial tissue. This type of cavitation is due to oedema, which produces a spongy state leading to liquefaction, and must be distinguished from true softening which results from necrosis of the interstitial tissue. This is characteristically absent in selective parenchymal necrosis and only follows more severe degrees of anoxia, which would be incompatible with life if the whole brain were subjected to them, but which may be encountered in restricted areas in lesions due to obstruction of arteries.

While the acceptance of a causal relationship between epileptic convulsions and brain damage is slowly gaining ground, we know of only one other example of severe damage to the brain following convulsions, which developed some days after an apparently trivial injury to the head, in a nonepileptic subject. This example is Scholz's (1951) case, which concerned a boy who was hit on the right eye while boxing with another boy. Epilepsy followed three days later and became more severe with a progressively heavier neurological disability during the next nine years. His symptoms were predominantly unilateral, and at necropsy there was atrophy of the corresponding cerebral hemisphere only.

The connexion between the trivial blow to the head and the disastrous epileptic convulsions which followed remains quite obscure in both Scholz's case and our own. Scholz's case, as well as that of Meyer et al., shows the end-results of selective parenchymal necrosis. These resemble closely the diffuse cerebral sclerosis with ulegyria described by one of us (Woolf, 1955) as the morphological basis of acute infantile cerebral diplegia. However, the sclerosis in the latter condition occupies a definite territory of venous drainage.

It is hoped that this case report will impress upon all those who have to treat cases of status epilepticus, whether following trauma to the head or not, the importance of minimizing anoxia during the attack, particularly by the maintenance of a free respiratory air-way.

\section{Summary and Conclusions}

The case is reported of a boy who received a trivial blow on the head, and, after a lucid interval of 24 hours, suffered a series of epileptic attacks followed by deepening unconsciousness and death.

During one of the more severe attacks amounting to status epilepticus, there was evidence of embarrassed respiratory function and accumulation of bronchial secretion.

Neurosurgical investigation and subsequent postmortem examination showed that there was no intracranial haemorrhage or other naked-eye finding to explain the deepening unconsciousness.

Histological examination of the brain showed widespread, though asymmetrical, cerebral degeneration typical of anoxia.

The possibility of epileptic convulsions, whether traumatic or otherwise, causing anoxic damage to the brain is emphasized.

The role of respiratory embarrassment during status epilepticus in producing this anoxia is stressed.

The connexion between the trivial head injury and the convulsions remains obscure, but a similar case is recorded in the literature.

Our thanks are due to Professor J. H. Dible for his kindness in advising us on the sections of the liver, and to Mr. K. J. Kirkham for giving us access to his clinical notes on this case.

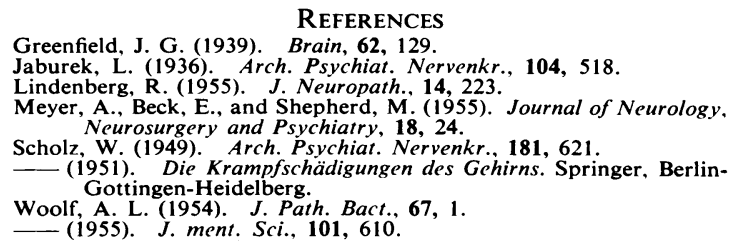

